\title{
Quantification of Ventricular Repolarization Dispersion on the Electrocardiogram by means of $T$ Wave Duration
}

\author{
PD Arini ${ }^{1}$, ER Valverde ${ }^{2}$, GC Bertrán ${ }^{3}$, P Laguna $^{4}$ \\ ${ }^{1}$ Argentine Institute of Mathematics, CONICET, Buenos Aires, Argentine \\ ${ }^{2}$ School of Engineering, University of Buenos Aires, Buenos Aires, Argentine \\ ${ }^{3}$ Institute of Medical Research Dr A Lanari, University of Buenos Aires, Buenos Aires, Argentine \\ ${ }^{4}$ Aragón Institute of Engineering Research, University of Zaragoza, Zaragoza, Spain
}

\begin{abstract}
Increased ventricular repolarization dispersion $(V R D)$ has been associated with risk of malignant ventricular arrhythmia. Several ECG parameters have been propose to quantify the $V R D$, which do not have definitive validation. The objective is to find and validate markers which represent the VRD more accurately. We hypothesized that $V R D$ increment, is reflected by T-wave widening. T-wave duration $\left(T_{w d}\right)$ is therefore proposed as a index to quantify $V R D$. The $T_{w d}$ and the so call T-wave residuum $\left(T_{w r}\right)$ have been computed. An isolated rabbit heart model was used. Global VRD, at all myocardium regions, was induced by supplying $d$ Sotalol and by premature ventricular stimulation (PVS). $T_{w d}$ showed significant increase with $V R D$ increase (78.0 \pm 10.3 vs $133.6 \pm 29.6 \mathrm{~ms}$ with $\mathrm{d}$-Sotalol and $95.2 \pm 7.9$ vs $118.5 \pm 15.7$ ms with $P V S$ ) while $T_{w r}$ did not change for this global VRD increase.
\end{abstract}

\section{Introduction}

Experimental and clinical studies have shown a link between ventricular repolarization dispersion $(V R D)$ and severe ventricular arrhythmia and/or sudden death, $V R D$ being a marker of cardiac risk [1]. The QT interval $(Q T)$ is used to quantify ventricular repolarization $(V R)$. $Q T$ dispersion $\left(Q T_{d}\right)$, measured as the difference between the maximum and minimum $Q T$ values of the standard 12-lead ECG, has also been proposed as an index to assess $V R D . Q T_{d}$ was proposed as a marker of the ventricular repolarization spatial dispersion, considering that each ECG lead records the local activity of different myocardium areas. To accomplish that, a Langendorff heart preparation was developed [2]. In that work, it was shown that the $Q T_{d}$ and JT dispersion showed a significant correlation with the dispersion of $A P D_{90}$ as well as with the dispersion of recovery time. Moreover, this fact was confirmed on patients, with a high correlation between the 24-hour $Q T_{d}$ and the intracardiac monophasic action potential [3]. However, the relationship between $Q T_{d}$ and $V R D$ is a subject of controversy due to the existence of both technical and theoretical problems regarding the measurement of the $Q T_{d}$. Several studies suggest that the result of the measurement might be affected by inaccuracies in the determination of the T-wave end and/or being the consequence of different projections of the electrical cardiac vector on the body surface leads, rather than $V R D$ [4]. The $Q T_{d}$ measured on the standard 12-lead ECG was compared with the one measured on synthesized 12 leads from the orthogonal XYZ leads [4], which leaves out the effects of regional heterogeneity. No differences between the two approaches were found. Another study showed that $Q T_{d}$ is significantly different between patients with a wide and a narrow T-wave loop. Moreover a correspondence was observed between the $Q T_{d}$ and the T-wave loop morphology. Recently [5], Singular Value Decomposition (SVD) of the ST-T complex, considering the three first components as the dipolar cardiac electrical vector representation $\left(c_{d}\right)$ and comparing them with the non-dipolar components $\left(c_{n d}\right)$, which were related to the myocardium local heterogeneity, has being proposed as $V R D$ index by the so call $T_{w r}$ index. Variations among different clinical groups as well as a low correlation with the $Q T_{d}$ were observed.

The present work aims at finding $V R D$ indexes on the surface ECG that quantify directly $V R D$. In order to achieve this purpose, an isolated In-Vitro rabbit heart model with several recording electrodes was used, on which $V R D$ was induced artificially. The ECG were analyzed at the signal resulting from summation of the absolute ECG value in 40 lead. The $T_{w r}$ from $S V D$ of the 40 lead were also analyzed. We want to validate the hypothesis that $T_{w r}$ do not show $V R D$ globally generated (in the whole myocardium) and that the $T_{w d}$ better reflects the the $V R D$. 


\section{Methods}

\subsection{Experimental model}

The experimental model consists of an In-Vitro system which records the electrical activity of an isolated rabbit heart beat-by-beat. The recording chamber, which simulates the animal's thorax has $7 \mathrm{~cm}$ diameter by 7 $\mathrm{cm}$ high. It has 40 recording electrodes homogeneously distributed in a 5 rows $\times 8$ columns array (Figure 1). The distance between electrodes is $10 \mathrm{~mm}$ and the angular distance is $45^{\circ}$, the electrodes having the Wilson Central Terminal point as reference. New Zealand white male rabbits $(2.8-3.8 \mathrm{Kg}$.) were used $(\mathrm{n}=20)$. The heart was removed and mounted on a vertical Langendorff apparatus. It was then immersed in the chamber with Tyrode solution and cannulized and perfused through the aorta with the same solution kept at $38^{\circ} \pm 0.5^{\circ} \mathrm{C}$, and bubbled with $\mathrm{O}_{2}$, with a flow of $700-900 \mathrm{ml} / \mathrm{h}$ and a pressure of $70 \mathrm{mmHg}$.

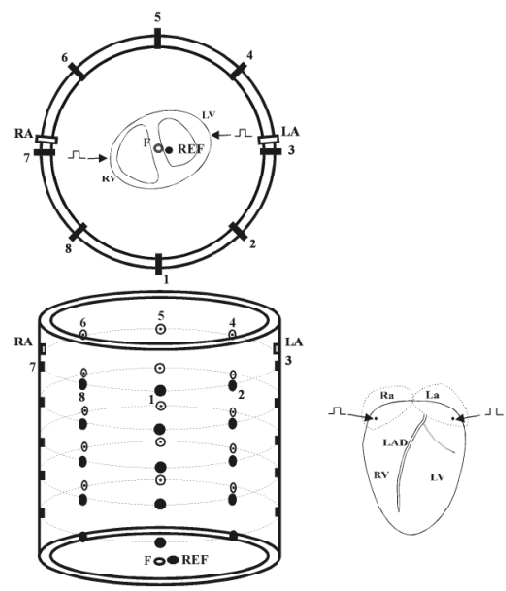

Figure 1. In Vitro system. Superior and frontal views showing the $5 \times 8$ matrix electrode plus the standard lead F, LA, RA and REF. It is also shown at the superior panel the stimulation site for right and left ventricle. The inferior right panel shows for left atrium (LA), right atrium (RA) and the left descending artery (LAD).

Two mechanisms were used in order to generate increased $V R D$. In the first one the increase was achieved by supplying d-Sotalol and in the other one by premature ventricular stimulation $(P V S)$. For the first protocol Tyrode solution was perfused for 30 minutes, this period being the control prior to d-Sotalol supply $\left(C_{D S}\right)$, after d-Sotalol solution was added and perfused $(60 \mathrm{mM})$ to generate increased dispersion $\left(D_{D S}\right)$. Both during $C_{D S}$ and $D_{D S}$ the ECG was recorded and the relevant variables measured. For the hearts being subject to the second protocol the heart was stimulated from the ventricles, in some cases from right ventricle $(R V)$ and in others from left ventricle $(L V)$ at basal frequency during a train of 49 beats. After that train, at beat number 50, a premature beat was generated at a coupling interval corresponding to the effective refractory period (Erp) plus $5 \mathrm{~ms}$. Erp was estimated on each case prior to the $P V S$ operation. Again, the ECG was recorded during the process. The measurement of $48 t h$ and $49 t h$ beats were averaged as control measures prior to ventricular stimulation $\left(C_{P V S}\right)$. The premature beat was elicited in order to generate dispersion paced to $R V$ or $L V\left(D_{P V S}\right)$. In both protocols the sinus node was crushed and an artificial pacemaker was used. In the d-Sotalol protocol the heart was stimulated from the right auricle at a basal frequency of $500 \mathrm{~ms}$ for $C_{D S}$ and $D_{D S}$ (10 cases). In the $P V S$ protocol the stimulation was done from the $R V$ (5 cases) and from the $L V$ (5 cases), at a basal frequency of $400 \mathrm{~ms}$ for control and at a frequency equal to the $E_{r p}+5 \mathrm{~ms}(165 \pm 7.5 \mathrm{~ms}$ for $R V$ stimulation and $170 \pm 12.9 \mathrm{~ms}$ for $L V$ ).

\subsection{Data acquisition and signal processing}

The ECG was acquired with instrumentation amplifiers with a gain factor of 1000, and a band width of 0.05-300 $\mathrm{Hz}$. They were digitalized at $1 \mathrm{Khz}$ and 12-bit resolution. When necessary, a band-stop filter to remove $50-\mathrm{Hz}$ was used. The baseline movement was compensated with a cubic spline algorithm. Once the heart electrical activity became stable (arrhythmia-free, ischemia-free and with no frequency variations), the beats corresponding to the first row were recorded, after which the same procedure was applied to the remaining rows. After selection and segmentation of a $i_{r} t h$ beat from each row $r(r=1, \ldots, 5)$ a signal, $x_{l, r}(n)$, for each derivation $l(l=1, \ldots 8)$ is obtained:

$$
\mathbf{x}_{l, r}=\left[x_{l, r}(0), \ldots, x_{l, r}(N-1)\right]^{T} .
$$

In order to quantify the $V R$ the following fiducial points were detected: T-wave onset $\left(T_{o}\right)$, T-wave end $\left(T_{e}\right)$ and then the $x_{l, r}(n)$ signals extend from from the $T_{o}$ to the $T_{e}$. The five $i_{r} t h$ selected beats are aligned, assuming that they represent simultaneous electrical activity, since electrical stability through all the registers has been met. The alignment was made with the QRS complex maximum upstroke slope. Selecting a beat implies taking a window (ranging from 300 to $400 \mathrm{~ms}$ ) including the repolarization phase. For each experimental environment $\left(C_{D S}, D_{D S}\right.$, $C_{P V S}, D_{P V S}, 40$ ECG lead recordings were obtained which were considered representative of the same heart electrical activity measured with different electrodes and beats $i_{1} t h, \ldots, i_{5} t h$ arranged in matrix

$$
\mathbf{X}_{\mathbf{i}}=\left[\mathbf{x}_{\mathbf{1}, \mathbf{1}}, \ldots, \mathbf{x}_{\mathbf{8}, \mathbf{1}}, \mathbf{x}_{\mathbf{1}, \mathbf{2}}, \ldots, \mathbf{x}_{\mathbf{8}, \mathbf{5}}\right] .
$$

From the matrix $\mathbf{X}_{\mathbf{i}}$, the parameters described in next subsections were measured. 


\subsubsection{Dipolar analysis}

The matrix $\mathbf{X}_{\mathbf{i}}$ is subjected to $S V D$ [6]. The singular values, $\sigma_{j}(j=1, \ldots, 40)$, are ordered such that $\sigma_{1} \geq \sigma_{2} \geq$ $\sigma_{3} \geq \ldots . \geq \sigma_{40} \geq 0$, and the $c_{d}$ and $c_{n d}$ are defined as

$$
c_{d}=\sum_{i=1}^{3} \sigma_{i}^{2} \quad \text { and } \quad c_{n d}=\sum_{i=4}^{40} \sigma_{i}^{2},
$$

The $S V D$ transfers the $\mathbf{X}_{\mathbf{i}}$ matrix ECG signal to a minimal orthogonal space. The first three components define the $c_{d}$, dipolar cardiac electrical vector component containing around $98 \%$ of the total energy, while the remaining ones grouped at $c_{n d}$, represent the ECG contribution which cannot be represented through the dipolar model. The $c_{n d}$ are due to regional heterogeneities or noise during acquisition. In order to quantify the relative contribution of the $c_{n d}$, the $T_{w r}$ index is calculated as:

$$
T_{w r}=\frac{c_{n d}}{c_{d}+c_{n d}}
$$

\subsubsection{Temporal analysis}

In addition to $T_{o}$ and $T_{e}$ points the T-wave peak location $\left(T_{p}\right)$ and T-wave width, $T_{w d}=T_{e}-T_{o}$, were computed from two ECG derived signals:

- The signal, $\mathbf{x}_{\mathbf{M}_{e}}$, obtained from the module of the first three eigenvectors of the SVD decomposition, $\left(\mathbf{x}_{\mathbf{e}_{\mathrm{j}}}\right.$, $j=1,2,3)$ :

$$
x_{M_{e}}(n)=\sum_{j=1}^{3}\left|x_{e_{j}}(n)\right|
$$

- The signal, $\mathbf{x}_{\mathbf{M}_{\mathbf{s}}}$, from the module of raw ECG:

$$
x_{M_{s}}(n)=\sum_{l=1}^{8} \sum_{r=1}^{5}\left|x_{l, r}(n)\right| .
$$

On these signals, denoted compactly by $\mathbf{x}_{\mathbf{P}}, P \in$ $\left\{M_{e}, M_{s}\right\}$ the following parameters are measured: $\mathrm{T}$ wave onsets: $T_{o}^{P}$; T wave ends: $T_{e}^{P}$; $\mathrm{T}$ wave peak: $T_{p}^{P}$; and $\mathrm{T}$ wave widths: $T_{w d}^{P}=T_{e}^{P}-T_{o}^{P}$.

All these time measures, $T_{o}^{P}, T_{e}^{P}$, and $T_{p}^{P}$, are refered to the QRS onset and express in ms. The fiducial points were detected by using a threshold-based algorithm on the differentiated signal [7]. Once the maximum and minimum of the differentiated signal were detected (maximum slope points) a threshold $K$ was established to detect the $T_{o}$ $\left(K_{o}=0.8\right)$ and and $T_{e}\left(K_{e}=0.2\right)$. The $T_{p}$ position was determined by the zero-crossing on the differentiated signals.

\subsection{Statistical analysis}

In order to determine whether the proposed parameters do discriminate control situations from those with increased $V R D$ (generated with d-Sotalol or $P V S$ ), the Wilcoxon test was used. A non-parametrical test was chosen since the distribution of variables to be compared was unknown.

\section{Results}

Table 1 presents the mean values \pm SD obtained by measuring $T_{o}^{M_{s}}, T_{e}^{M_{s}}, T_{p}^{M_{s}}, T_{w d}^{M_{s}}$ and by computing $T_{w r}$ on the signal segment delimited by $T_{e}^{M_{e}}$ and $T_{o}^{M_{e}}$. Results in control beats and in increased $V R D$ beats (generated by $P V S$ and d-Sotalol) are shown. In the case of $P V S$ it can be observed that the variables $T_{o}^{M_{s}}$ and $T_{p}^{M_{s}}$ are reduced by $20 \mathrm{~ms}$ with respect to the control, both differences being statistically significant. On the other hand, the $T_{e}^{M_{s}}$ variable did not present statistically significant differences between control and $P V S$, while $T_{w d}$ increased by $20 \mathrm{~ms}$, this difference being statistically significant. When the $V R D$ is increases by d-Sotalol, it can be observed that the $T_{o}^{M_{s}}$ and $T_{e}^{M_{s}}$ variables change significantly with a shortening of $20 \mathrm{~ms}$ and a lengthening of $35 \mathrm{~ms}$, respectively. On the other hand, the $T_{p}^{M_{s}}$ variable did not present statistically significant differences between control and d-Sotalol while the $T_{w d}^{M_{s}}$ variable increased significantly as the T-wave widened by $55 \mathrm{~ms}$. Furthermore, the $T_{w r}$ did not change between the control and the increased $V R D$ by means of any of the mechanisms to generate $V R D$. Figure 3 shows ECG recordings in one control and a $V R D$ increased case.

Table 1. Results given in ms for time variables. ${ }^{(*)}$ denotes significative difference $(p<0.006)$

\begin{tabular}{|l||c|c|c|c|}
\hline & $C_{P V S}$ & $D_{P V S}$ & $C_{D S}$ & $D_{D S}$ \\
\hline$T_{o}^{M_{s}}$ & $123 \pm 15$ & $99 \pm 18^{(*)}$ & $137 \pm 12$ & $115 \pm 34^{(*)}$ \\
\hline$T_{e}^{M_{s}}$ & $220 \pm 14$ & $217 \pm 16$ & $215 \pm 18$ & $249 \pm 25^{(*)}$ \\
\hline$T_{p}^{M_{s}}$ & $163 \pm 12$ & $142 \pm 13^{(*)}$ & $169 \pm 13$ & $165 \pm 27$ \\
\hline$T_{w d}^{M_{s}}$ & $95 \pm 7$ & $118 \pm 15^{(*)}$ & $78 \pm 10$ & $133 \pm 29^{(*)}$ \\
\hline$T_{w r}$ & $0.27 \pm 0.21$ & $0.19 \pm 0.08$ & $0.18 \pm 0.16$ & $0.26 \pm 0.28$ \\
\hline
\end{tabular}

\section{Discussion and conclusions}

During cardiac depolarization and repolarization, local effects may appear which cannot be well represented by means of a dipolar model, this effect being more marked in regional pathological conditions. The $T_{w r}$ is an index which reflects the $V R D$ local heterogeneity [8] through the $c_{n d}$. In the present experimental model, the $T_{w r}$ index 


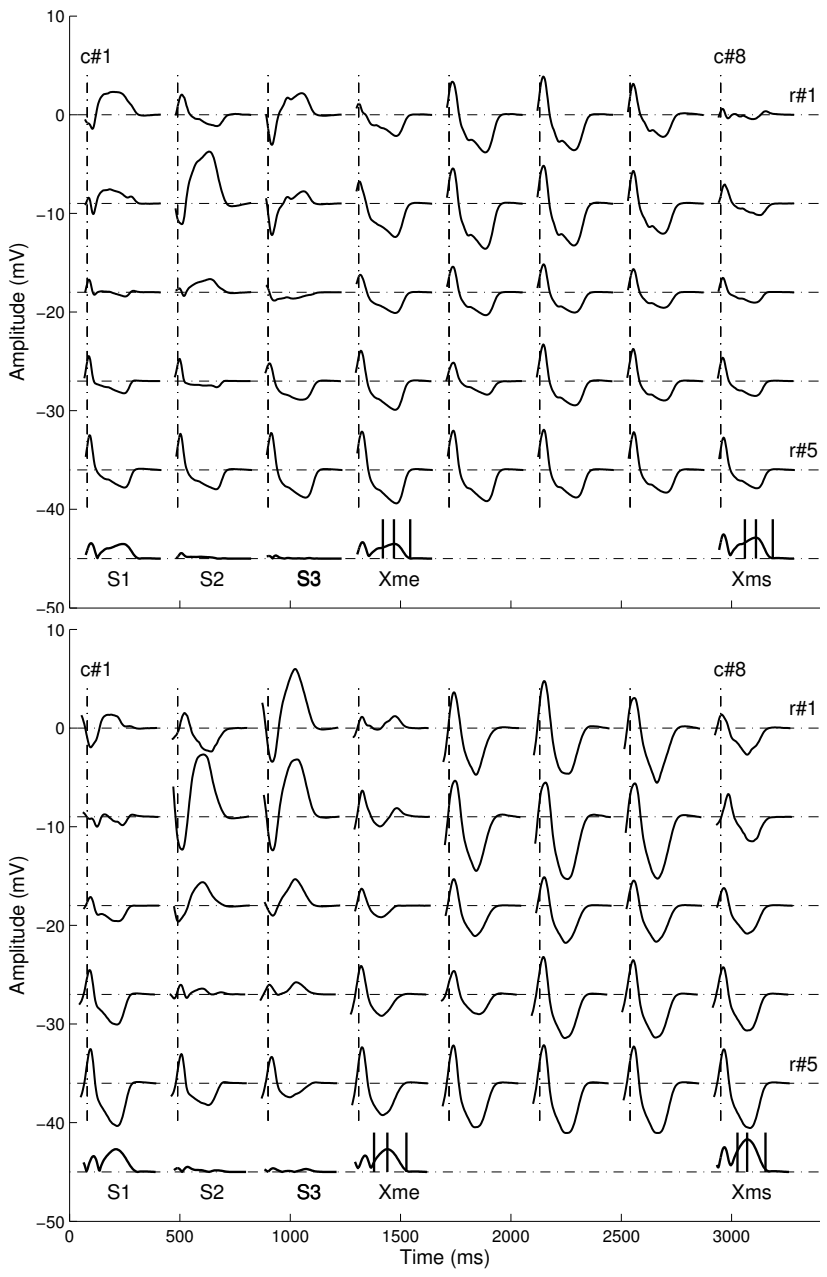

Figure 2. Top panels: the $40 \mathrm{ECG}$ recordings in a $C_{P V S}$ (LV). Bottom panels their corresponding for $D_{P V S}$. Over plotted are the three first components when SVD is applied $\left(S_{1}, S_{2}\right.$ and $\left.S_{3}\right)$, and the reconstructed module signals $x_{M_{s}}$ and $x_{M_{e}}$ with the fiducial points overmark

did not detect increased $V R D$, probably due to the fact that both the premature beats and the supply of d-Sotalol generated $V R D$ homogenously distributed throughout the whole myocardium, and then still well represented by the dipolar model. T-wave duration increased as $V R D$ augmented. This $T_{w d}^{M_{s}}$ increase indicates a differential shortening or lengthening of the AP in some myocardium areas measuring the time span at which different APs are ending and therefore it could be considered an expression of the AP duration dispersion. Other variables also showed changes: $T_{o}^{M_{s}}$ and $T_{p}^{M_{s}}$ shortening in $P V S$, indicating dispersion generated by early ending AP in $P V S$ situations; $T_{o}^{M_{s}}$ and $T_{e}^{M_{s}}$ decrease and increase, respectively, as d-Sotalol was supplied supporting the hypothesis that $d$-Sotalol generates both situation at different areas of the myocardium, early ending AP at some areas and delayed ending at other areas.

The hypothesis that increased $V R D$ implies a widening in the T-wave duration is corroborated by the present results, when $V R D$ is generated across the whole myocardium.

\section{Acknowledgements}

This work was supported by projects: TIC 2001-2167CO2-02 (MCYT), P075/2001 and "Grupos Consolidados" (CONSID-DGA), and by a personal grant to Pedro Arini from the "Programa de Movilidad Académica de la Asociación Universitaria Iberoamericana de Postgrado", AUIP 2002-2003, Spain.

\section{References}

[1] Kuo C, Munakata E, Reddy P, Surawicz B. Characteristics and possible mechanism of ventricular arrythmia dependent on the dispersion of action potential durations. Circulation 1983;67:1356-1367.

[2] Zabel M, Portnoy S, Franz. Electrocardiographic indexes of dispersion of ventricular repolarization an isolated heart validation. JACC 1995;25(3):746-752.

[3] Zabel M, Lichtlen PR S, Haverich A, Franz M. Comparison of ECG variables of dispersion of ventricular repolarization with direct myocardial repolarization measurements in the humans heart. J Cardiovasc Electrophysiol 1998;9:12791284.

[4] P.W. Macfarlane SM, Rodger C. Influence of lead selection and population on automated measurement of QT dispersion. Circulation 1998;98.

[5] Malik M, Acar J, Yi B, Yap Y, K H, Camm J. QT dispersion does not represent electrocardiographics heterogeneity of ventricular repolarization. $\mathrm{J}$ of Cardiovasc Electrophysiol 2000;11(8):835-843.

[6] Acar B, Koymen H. SVD-based on-line exercise ECG signal orthogonalization. IEEE Trans on Biomed Eng 1999;46(3).

[7] Laguna P, Thakor N, Caminal P, R J. New algoritm for QT interval analysis in 24-hours holter ECG: perfomance and applications. Med Biol Comput 1990;28(3):67-73.

[8] Smetana P, Batchvarov V, Hnatkova K, Camm A, Malik M. Ventricular gradient and nondipolar repolarization components increase at higher heart rate. AJP Heart Circ Physiol 2004;286:H131-H136.

Address for correspondence:

Pedro David Arini

Instituto Argentino de Matemática, Saavedra 15 piso 3 (1083)

Buenos Aires, Argentina.

pedroarini@fibertel.com.ar 